\title{
Explosion Math: um jogo digital como recurso na aprendizagem de Matemática
}

Bruna R. Takita*, Jackson R. Santana**, Pedro M. de Souza***

\section{Resumo}

Este artigo tem como objetivo apresentar o desenvolvimento e a aplicação de um jogo digital educacional que aborda conteúdos específicos da Matemática dos anos finais do ensino fundamental I, com foco no $5^{\circ}$ ano. Explosion Math, inspirado no jogo clássico Bomberman, foi desenvolvido utilizando o motor gráfico Unity 3D e pretende auxiliar o ensino de matemática de forma diferenciada e dinâmica. O jogo propõe aos alunos uma viagem com o astronauta Albert, um garoto apaixonado por Matemática, que, ao descobrir a existência de planetas matemáticos, embarca em uma aventura espacial. A missão de Albert é explorar os planetas Numérico, Geométrico e Grammy (grandezas e medidas), a fim de obter o conhecimento presente em cada um deles.

Palavras-chave: Ensino de matemática. Explosion Math. Jogos digitais.

\section{Introdução}

A Matemática é considerada, por muitos alunos, como uma disciplina complexa e enfadonha. Isso reflete em alunos desmotivados a aprender os conteúdos dessa disciplina e, consequentemente, gera resultados insatisfatórios em Matemática nos sistemas de avaliação da educação brasileira (CHAS, 2014; ARAÚJO; COSTA; RIBEIRO JÚNIOR, 2016). De acordo com a Organization for Economic Cooperation and Development (OECD), os resultados do Programme for International Student Assessment (Pisa) indicam que a motivação e a confiança dos estudantes em si mesmos são essenciais para que aproveitem ao máximo seu potencial (ORGANISATION FOR

Bacharel em Sistemas de Informação. Ex-estudante da Universidade Federal de Viçosa. E-mail: brunatakita@ hotmail.com

* Bacharel em Sistemas de Informação. Estudante da Universidade Federal de Viçosa. E-mail: jack.rsantana@ gmail.com

**** Mestre. Professor na Universidade Federal de Viçosa. E-mail: profpedromoises@gmail.com 
ECONOMIC CO-OPERATION AND DEVELOPMENT, 2013). Dornelles (2005), Sartori e Duarte (2014) também defendem que o objetivo do educador não se restringe a ensinar o conteúdo proposto, ele também deve motivar, incentivar e estimular o desejo de aprender do aluno.

Analisando os resultados de sistemas nacionais e internacionais de avaliação da educação básica, como, por exemplo, o Pisa e a Prova Brasil, é possível evidenciar que a educação brasileira não está em sua melhor condição (ARAÚJO; COSTA; RIBEIRO JÚNIOR, 2016). Os dados do Pisa (OECD, 2016) mostram uma queda do Brasil no ranking mundial desde 2009. Na última edição da prova, em 2015 , o país ficou na $63^{\mathrm{a}}$ posição em Ciências, na 59aㅡ em Leitura e na $66^{\mathrm{a}}$ colocação em Matemática, numa escala de 70 países. Segundo os resultados da Prova Brasil (QEDU, 2015), realizada em 2015, somente $50 \%$ dos alunos até o $5^{\circ}$ ano da rede pública aprenderam o adequado na competência Leitura e Interpretação de Textos, e, em Matemática, o índice foi de somente $39 \%$.

Nesse contexto, os jogos digitais entram em cena como soluções tecnológicas, associando o entretenimento à capacidade de ensinar, fazendo com que o aluno se sinta envolvido e motivado a aprender por meio de seu caráter lúdico (JACOBSEN; SPEROTTO, 2013). A utilização de jogos possibilita a aprendizagem de forma mais visual e dinâmica, associando a matemática da sala de aula à matemática do cotidiano e apresentando o conhecimento de forma mais tangível (BRITES et al., 2014; CHAS, 2014).

Portanto, o jogo digital Explosion Math, apresentado neste artigo, visa a auxiliar e a complementar o processo de ensino da matemática nos anos finais do ensino fundamental I, com foco no $5^{\mathrm{o}}$ ano, abordando conteúdos específicos, como geometria, operações numéricas, grandezas e medidas. Explosion Math, inspirado no jogo clássico Bomberman, pretende estimular o interesse dos alunos em aprender matemática, de forma a promover um ensino diferenciado e dinâmico. Nas próximas seções, apresentam-se a fundamentação teórica, trabalhos relacionados, a metodologia, a descrição do jogo, os resultados e, por fim, as considerações finais.

\section{Fundamentação teórica}

$\mathrm{O}$ avanço da tecnologia e sua intensa utilização pela população em geral, aliados à evolução das formas de se construir conhecimentos, proporcionam um cenário adequado para o surgimento dos jogos digitais enquanto ferramentas de apoio à educação, porque os jogos digitais podem auxiliar o desenvolvimento de conhecimento e habilidades cognitivas, como a resolução de problemas, o pensamento estratégico, a tomada de decisão, entre outros. Dessa forma, facilita-se 
uma compreensão mais profunda do conteúdo abordado (ALVES; LOBATO; BITTAR, 2013; BROM; PREUSS; KLEMENT, 2011).

De acordo com Savi e Ulbricht (2008), os jogos digitais educacionais fornecem diversos benefícios ao processo de ensino-aprendizagem, dentre eles, o efeito motivador, o aprendizado por descoberta, a experiência de novas identidades, a socialização, a coordenação motora e o comportamento expert: experiências de novas identidades, pois os jogos oferecem aos estudantes a vivência de experiências em outros mundos e realidades por meio da imersão; socialização, uma vez que aproxima os jogadores competitiva ou cooperativamente; e comportamento expert, pois os jogos podem ter potencial de tornar seus jogadores experts nos conteúdos abordados por meio do envolvimento desses jogadores com os desafios educacionais propostos.

\section{Trabalhos relacionados}

No trabalho de Menezes e Roza (2016), foi desenvolvido um aplicativo baseado em figuras e sons, nomeado Genius Math. O aplicativo visa a apoiar o ensino da matemática na educação infantil e foi desenvolvido utilizando a linguagem de programação Java. Os conceitos matemáticos explorados neste aplicativo são os números, as formas geométricas e as operações de adição e subtração.

Figura 1 - Genius Math

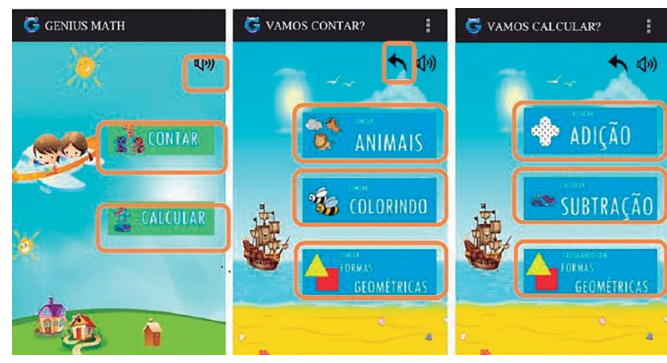

Fonte: Menezes e Roza (2016).

O aplicativo Genius Math se assemelha ao Explosion Math, pois ambos visam a auxiliar e a complementar o processo de ensino de matemática na educação infantil utilizando a tecnologia. Entretanto, Genius Math propicia o aprendizado da criança sem a dinamicidade dos jogos digitais. Explosion Math, por sua vez, promove a assimilação dos conceitos matemáticos abordados por meio de seu caráter lúdico e dinâmico.

Por meio da pesquisa bibliográfica, outros trabalhos relacionados foram analisados, como os desenvolvidos por Moraes e Colpani (2016) e Nakagawa et al. (2016).

\section{Metodologia}

A elaboração do jogo educativo Explosion Math se embasou no processo ágil de desenvolvimento de Sistemas de Realidade Virtual, que é baseado nos 
modelos iterativo e evolucionário (TORI; KIRNER; SISCOUTTO, 2006; MATTIOLI et al., 2009; SOMMERVILLE; SAWYER, 1997). Esse processo é caracterizado pela sua natureza evolutiva, pelo acolhimento de alterações e pela melhor aceitação de sistemas com participação ativa de usuários finais no processo de desenvolvimento (MATTIOLI et al., 2009). Tais características são extremamente adequadas ao desenvolvimento de jogos educativos, visto que existem grandes possibilidades de mudanças, devido a intensa interação entre os atores envolvidos no projeto.

O processo ágil de desenvolvimento utilizado é composto por cinco etapas, que podem ser utilizadas iterativamente, sendo elas: Análise de Requisitos, Projeto, Implementação, Avaliação e Implantação.

\section{Análise de Requisitos}

Nesta etapa do presente projeto, foram obtidos o escopo, a storyboard e os requisitos básicos do projeto. Por se tratar de um jogo educativo, que tem como objetivos auxiliar o ensino de matemática e não substituir o professor, esse último tem um papel importante e indispensável no desenvolvimento do jogo.

Sendo assim, por meio de informações coletadas em entrevista com uma profissional da área, foram possíveis estabelecer o conteúdo de ensino e a melhor forma de abordá-lo no jogo. $\mathrm{O}$ conteúdo de ensino abordado faz parte da matriz curricular do $5^{\circ}$ ano do ensino fundamental I e abrange os seguintes conceitos matemáticos: operações numéricas, geometria, grandezas e medidas. A abordagem do conteúdo de ensino no jogo foi realizada pelo conceito de associação. Sendo assim, o jogador deve associar e coletar as respostas corretas, de acordo com o objetivo de cada fase.

\section{Projeto}

Segundo Tori, Kirner e Siscoutto (2006), a etapa de Projeto consiste em colocar em prática os requisitos definidos e analisados na etapa anterior: são especificadas a arquitetura do sistema e as tecnologias a serem utilizadas no projeto, assim como a definição dos elementos e os objetos do ambiente virtual, detalhando características, comportamentos e interações entre eles.

Nesta etapa, foram definidas as tecnologias utilizadas para implementação do projeto: a Unity $3 \mathrm{D}$, por ser um motor gráfico que oferece suporte e diversos recursos para o desenvolvimento de jogos, possibilitando a criação de games em duas ou três dimensões; a programação em JavaScript ou C\#; a conexão com diversos tipos de bancos de dados; e a exportação do jogo para várias plataformas. 
Foram definidos também softwares, como Photoshop e Corel Draw, para criação de imagens e sprites gráficos em 2D. $\mathrm{E}$, para não sobrecarregar a Unity $3 \mathrm{D}$, um banco de dados externo, utilizando a biblioteca SQLite, foi implementado para o armazenamento de perguntas, alternativas e respostas corretas.

\section{Implementação}

A etapa de Implementação, de acordo com Tori, Kirner e Siscoutto (2006), consiste na utilização das tecnologias disponíveis e definidas na etapa de Projeto, para concretizar o sistema, abrangendo, assim, a construção das fases, a criação das imagens e dos sprites e a composição do cenário virtual.

Nesta etapa do projeto, foram desenvolvidos: os scripts contendo a lógica de programação do jogo, por meio da linguagem C Sharp (C\#); a construção das fases e a interface do jogo, por meio dos componentes da Unity 3D; a conexão com o banco de dados externo, por meio da biblioteca SQLite; e a criação das imagens e dos sprites gráficos em 2D, utilizando o Corel Draw.

\section{Avaliação}

A Avaliação pode ser feita de diferentes formas, como, por exemplo, por testes formais, estudos empíricos e observação informal, avaliando características como desempenho, usabilidade e eficácia (SOMMERVILLE; SAWYER, 1997; TORI; KIRNER; SISCOUTTO, 2006).

Ao longo do período de implementação, foram realizados testes nas funcionalidades do jogo, visando a verificar se ele atingia o objetivo proposto. Além disso, o jogo foi apresentado ao seu público-alvo, alunos dos anos finais do ensino fundamental I, na III Mostra de Jogos. ${ }^{1}$ Durante a atividade, foram aplicados questionários aos usuários, a fim de avaliar a usabilidade do jogo e coletar os resultados necessários.

\section{Implantação}

De acordo com Tori, Kirner e Siscoutto (2006), a etapa de Implantação está relacionada ao ambiente em que o sistema é inserido. Explosion Math, por se tratar de um jogo educativo, foi inserido em ambiente escolar.

Para que o jogo possa ser utilizado pelos interessados, sua versão final será disponibilizada no servidor da Universidade Federal de Viçosa, campus Rio Paranaíba.

\section{Descrição do jogo}

Explosion Math propõe aos alunos uma viagem com o astronauta Albert (avatar do jogo), um garoto apaixonado por matemática que, ao descobrir a existência de planetas matemáticos, 
embarca em uma aventura espacial, com a missão de explorar os planetas Numérico, Geométrico e Grammy (grandezas e medidas), a fim de obter o conhecimento presente em cada um deles, conforme a storyboard representada na Figura 2.

Figura 2 - Storyboard do jogo educativo Explosion Math

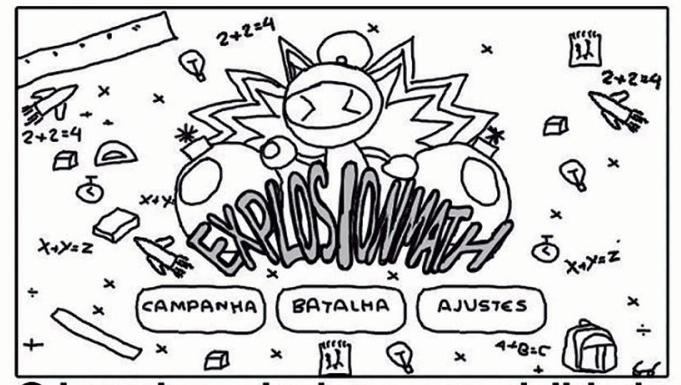

O jogador seleciona a modalidade.

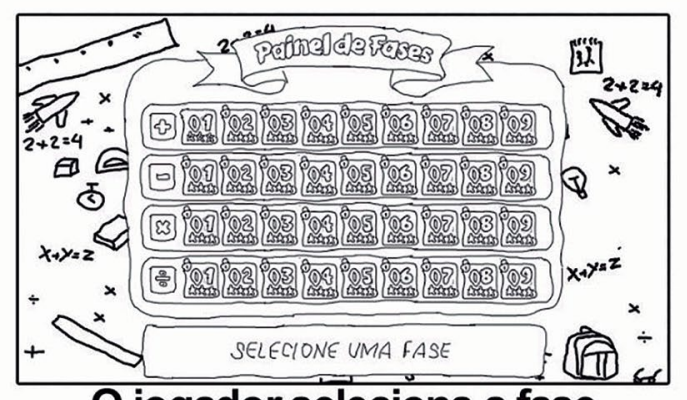

0 jogador seleciona a fase.

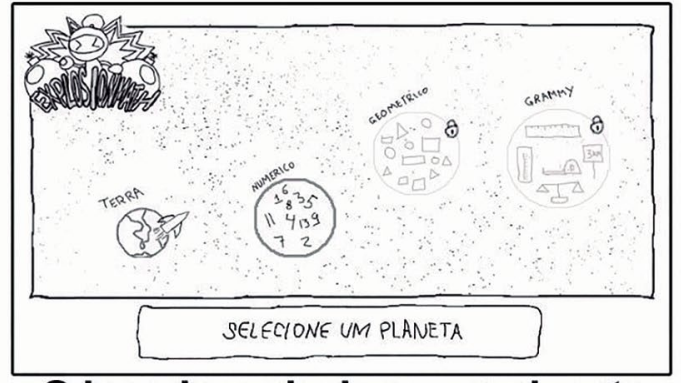

O jogador seleciona um planeta matemático.

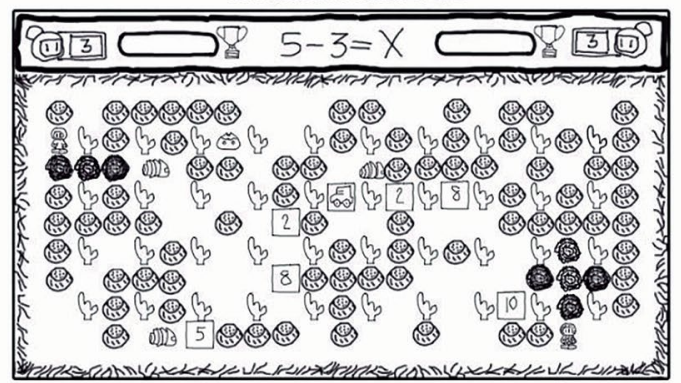

O jogador percorre o cenário coletando as respostas certas.

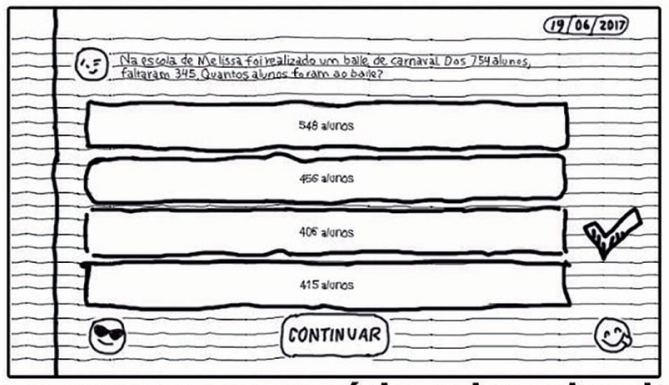

\section{Ao coletar um power up no cenário, o jogador deve responder corretamente uma pergunta para receber o benefício.}

Fonte: elaboração dos autores. 
O jogo é constituído pelas modalidades Campanha, Batalha e Área do Professor (Figura 3). As duas primeiras são destinadas principalmente aos alunos, $\mathrm{e}$ a última, ao professor.

Figura 3 - Tela de seleção de modalidade

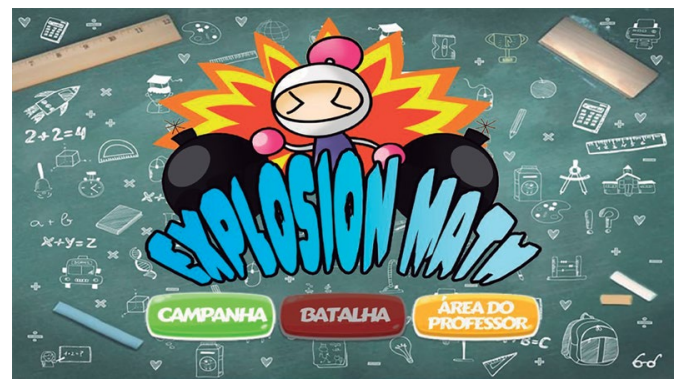

Fonte: elaboração dos autores.

Na modalidade Campanha, o jogador se aventura com Albert na missão de explorar os planetas matemáticos, enfrentando inimigos e coletando as respostas corretas referentes ao objetivo da fase em questão. A modalidade Batalha, ainda não implementada, permitirá que mais de um jogador embarque nessa aventura ao mesmo tempo, competindo para obter o conhecimento dos planetas. E a modalidade Área do Professor permite o gerenciamento de perguntas no banco de dados.

\section{Área do aluno}

Após selecionar a modalidade, o aluno/jogador deve selecionar o planeta a ser explorado, conforme a Figura 4. Inicialmente, somente o planeta Numérico está liberado para seleção, os demais são desbloqueados depois da conclusão da exploração do primeiro planeta.

Figura 4 - Tela de seleção de planeta

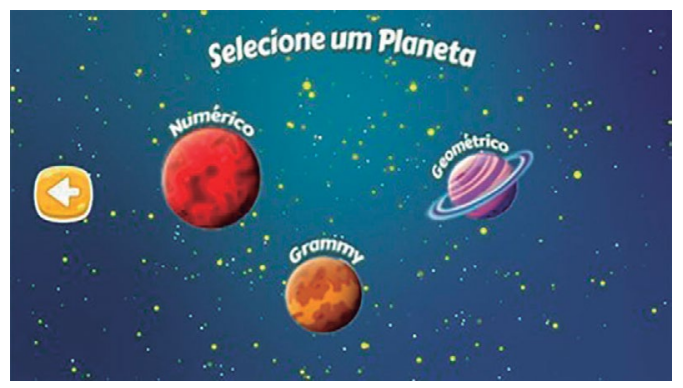

Fonte: elaboração dos autores.

O primeiro planeta é o Numérico, que contém o conhecimento em operações aritméticas, abordando adição, subtração, multiplicação e divisão. O segundo, o planeta Geométrico, é composto pelo conhecimento de formas geométricas e suas propriedades. E, por último, o planeta Grammy detém o conhecimento em grandezas e medidas, abrangendo medidas de tempo, capacidade, massa e comprimento.

Cada um dos planetas tem um painel de fases, em que o conteúdo geral é dividido em conteúdos específicos. $\mathrm{O}$ planeta Numérico é dividido em adição, subtração, multiplicação e divisão, conforme mostra a Figura 5. Assim como na seleção dos planetas, inicialmente somente a primeira fase é liberada para seleção, as demais serão desbloqueadas com a evolução do jogo. 
Figura 5 - Painel de fases

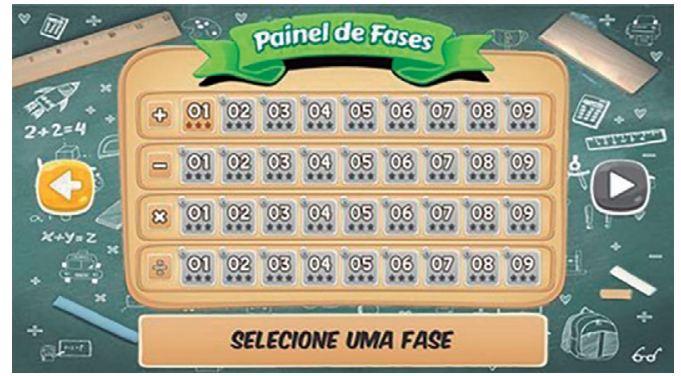

Fonte: elaboração dos autores.

O jogo contém uma barra de notificações (apresentada na Figura 6), que é composta pelo menu de objetivos e pela barra de progresso e mostra a quantidade de vidas. $O$ jogador inicia com três vidas, e essa quantidade é decrementada sempre que é atingido por inimigos ou explosões. Ao perder as três vidas, deve repetir a fase.

O menu de objetivos gera desafios aleatórios, baseados na área da matemática e na fase selecionada. Assim que uma resposta correta é coletada, um novo objetivo é gerado. E, por fim, a barra de progresso é responsável por contabilizar as respostas corretas coletadas pelo jogador. Ao completá-la, o jogador conclui a fase.

\section{Cenário e objetivo}

O cenário do jogo é constituído de obstáculos destrutíveis e indestrutíveis e de inimigos (Figura 6). Quando blocos são explodidos com bombas, possíveis respostas aparecem no cenário. $\mathrm{O}$ jogador deve discernir sobre o problema e coletar apenas as respostas corretas, de acordo com o menu de objetivos.
Figura 6 - Cenário no planeta Numérico e barra de notificações

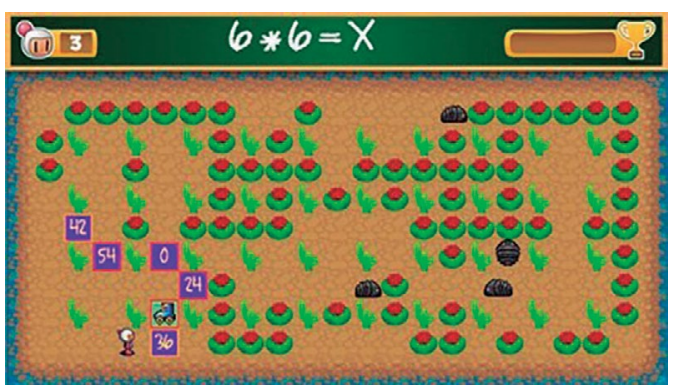

Fonte: elaboração dos autores.

Ao coletar um power up, o aluno/ jogador deve responder uma pergunta de matemática, selecionada aleatoriamente no banco de dados (Figura 7). Caso responda corretamente, recebe um benefício, que pode ser o aumento da quantidade de bombas, da velocidade ou do alcance das explosões.

Figura 7 - Tela de pergunta

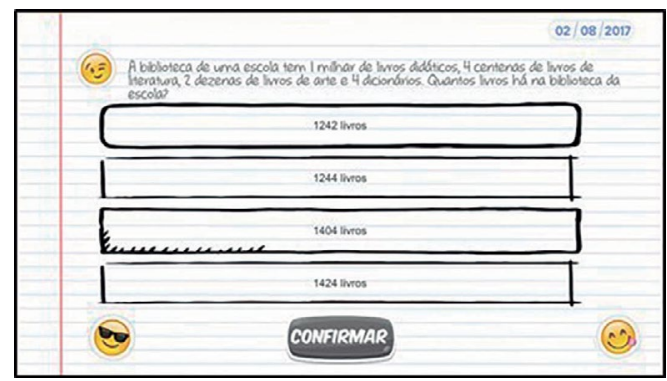

Fonte: elaboração dos autores.

Ao coletar 10 respostas corretas, o jogador conclui a fase; a tela representada na Figura 8 é exibida, e a próxima fase é liberada. 
Figura 8 - Tela de conclusão de fase

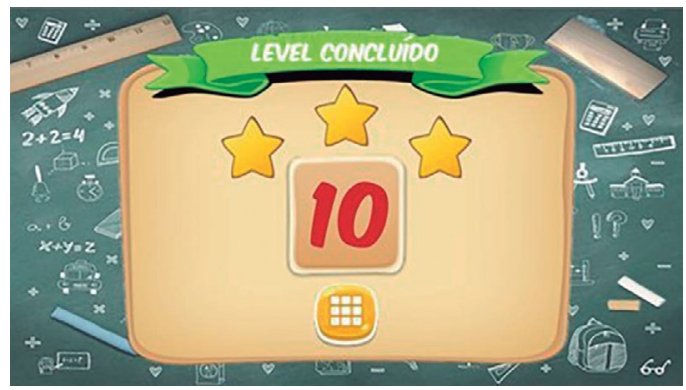

Fonte: elaboração dos autores.

\section{Área do Professor}

A Área do Professor somente poderá ser acessada por login e senha, visto que permite o acesso às perguntas do jogo. Nesta modalidade, o professor pode inserir, consultar e editar perguntas, conforme Figura 9. Na tela de edição, o professor também tem a opção de excluir a pergunta.

Figura 9 - Telas da Área do Professor (menu, inserir, consultar e editar)

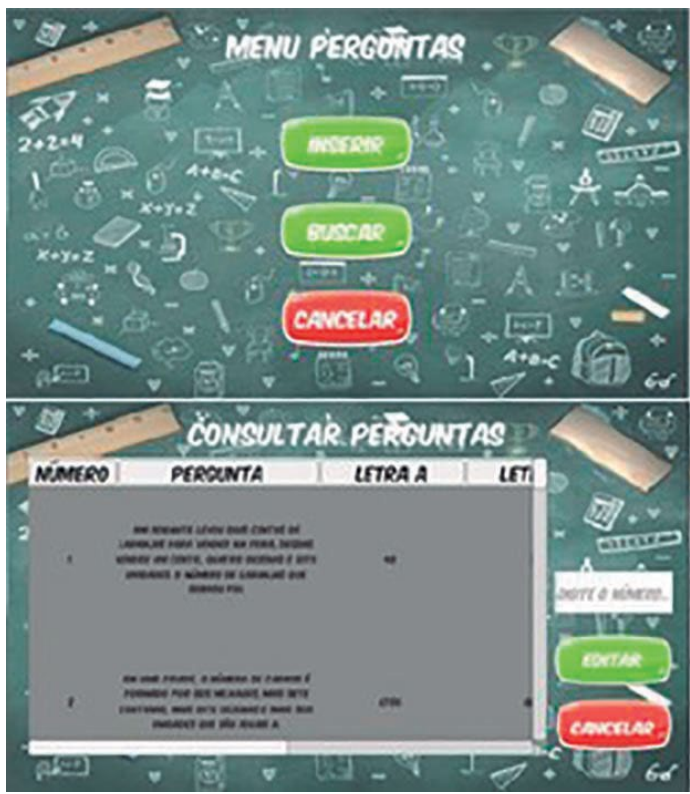

Fonte: elaboração dos autores.

\section{Resultados}

O protótipo do jogo Explosion Math foi apresentado ao seu público-alvo, alunos do ensino fundamental I, na III Mostra de Jogos, realizada na Universidade Federal de Viçosa, Campus Rio Paranaíba. Os

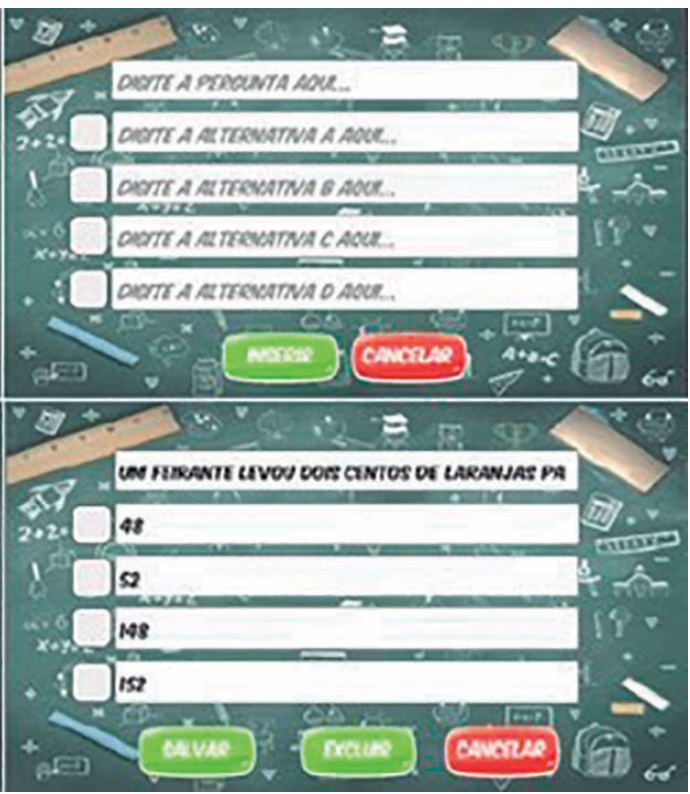

alunos testaram o jogo e responderam um questionário, a fim de avalia-lo. O questionário era composto de 14 perguntas, e os alunos responderam considerando uma escala de 1 a 5 , na qual 1 indicava muito insatisfeito e 5, muito satisfeito. Foram coletados 20 questionários. 
O Gráfico 1 apresenta os índices de duas das questões avaliadas no questionário. A primeira visou a verificar se o aluno foi estimulado a aprender com o jogo: $75 \%$ dos alunos atribuíram nota máxima à questão. $\mathrm{E}$ a segunda questionou-os sobre o potencial do jogo para auxiliar os estudos, $85 \%$ declararam que o jogo tem potencial.

Figura 10 - Resultado da avaliação do grau de satisfação

Você se sentiu estimulado a aprender Você acha que o jogo pode auxiliar com o jogo?
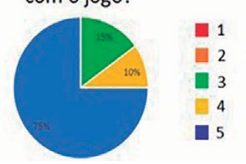
seus estudos?

Fonte: elaboração dos autores.

\section{Considerações finais}

De acordo com os resultados obtidos, Explosion Math teve boa aceitação na etapa de avaliação, uma vez que os alunos se mostraram muito satisfeitos com diversos aspectos do jogo e atribuíram nota máxima aos itens avaliados em grande parte das questões.

De modo geral, o jogo se apresentou como uma ferramenta potencial para auxiliar o ensino de matemática e, apesar dos pontos a serem aprimorados, cumpriu com o objetivo proposto de forma satisfatória. Como trabalhos futuros, pretendem-se finalizar o desenvolvimento do jogo e aprimorar os pontos diagnosticados na etapa de avaliação.

\section{Explosion Math: a digital game as a resource in Maths learning}

\section{Abstract}

This article has the objective of presenting the development and application of a digital game that approaches specific Math content of the final years of Elementary School, focused in the fifth year. Explosion Math, inspired in the classic game Bomberman, was developed using the Unity 3D graphic engine and intends to assist the Math teaching in a different and dynamic way. The game offer to the students a trip with the astronaut Albert, a boy who is in love with Math, that when discovers the existence of mathematical planets, gets on board of a spatial adventure. Albert's mission is to explore the Numerical, Geometrical and Grammy Planets, in order to obtain the knowledge that exists in each one of the planets.

Keywords: Explosion Math. Digital games. Maths teaching.

\section{Nota}

1 Evento que aconteceu na Universidade Federal de Viçosa, Campus Rio Paranaíba, no dia 05 de julho de 2017.

\section{Referências}

ALVES, R.; LOBATO, L. L.; BITTAR, T. J. Desenvolvimento de jogos digitais educativos e aplicação como ferramenta de ensino. In: ENCONTRO ANUAL DE TECNOLOGIA DA INFORMAÇÃO; SEMANA ACADÊMICA DE TECNOLOGIA DA INFORMAÇÃO, 2013. Santa Maria, 2013. Anais... Santa Maria, 2013. p. 271-274. 
ARAÚJO, J. P. P.; COSTA, G.; RIBEIRO JÚNIOR, J. G. Matematech: plataforma de apoio à aprendizagem de matemática nos anos iniciais do ensino fundamental. In: BRAZILIAN SYMPOSIUM ON COMPUTERS IN EDUCATION (SIMPÓSIO BRASILEIRO DE INFORMÁTICA NA EDUCAÇÃO-SBIE), 27., Uberlândia, 2016. Anais..., Uberlândia, 2016. p. 110.

BRITES, V. J. B. et al. O jogo no ensino de operações com números inteiros. In: SIMPEMAD -SIMPÓSIO EDUCAÇÃO MATEMÁTICA EM DEBATE, 1., Joinville, 2014. Anais..., Joinville, 2014. p. 330-330.

BROM, C.; PREUSS, M.; KLEMENT, D. Are educational computer micro-games engaging and effective for knowledge acquisition at high-schools? a quasi- experimental study. Computers \& Education, Oxford, v. 57, n. 3, p. 1971-1988, Nov. 2011.

CHAS, D. M. P. Matemática e atividades lúdicas: uma metodologia diferenciada. In: SIMPEMAD -SIMPÓSIO EDUCAÇÃO MATEMÁTICA EM DEBATE, 1., Joinville, 2014. Anais..., Joinville, 2014. p. 93-103.

DORNELLES, L. V. Infâncias que nos escapam: da criança na rua à criança cyber. São Paulo: Vozes, 2005.

JACOBSEN, D. R.; SPEROTTO, R. Jogos eletrônicos: um aprender lúdico e virtual para o ensino de matemática. In CONGRESSO INTERNACIONAL DE ENSINO DE MATEMÁTICA, 6., Canoas, 2013. Anais..., Canoas, 2013. p. 787-792.

MATTIOLI, F. E. et al. Uma proposta para o desenvolvimento ágil de ambientes virtuais. SBC. In: WRVA, Santos, 2009. Anais..., Santos, 2009. p. 230-235.

MENEZES, S.; ROZA, J. Genius math: uma aplicação mobile para auxiliar a aprendizagem da matemática na pré-escola. In: BRAZILIAN SYMPOSIUM ON COMPUTERS IN EDUCATION (SIMPÓSIO BRASILEIRO DE INFORMÁTICA NA EDUCAÇÃO-SBIE), 27. Uberlândia, 2016. Anais..., Uberlândia, 2016. p. 250 .
MORAES, I. G.; COLPANI, R. Desenvolvimento de um serious game, baseado na rerroupagem do clássico pac-man, para auxiliar no ensino de matemática básica. In: SBGAMES - SIMPÓSIO BRASILEIRO DE JOGOS E ENTRETENIMENTO DIGITAL, 15., São Paulo, 2016. Anais... São Paulo: Proceedings of SBGames. 2016. p. 758-761.

NAKAGAWA, W. et al. Geometric tower: game para auxílio no ensino lógico-matemático e espacial. In: WORKSHOPS DO CONGRESSO BRASILEIRO DE INFORMÁTICA NA EDUCAÇÃO, 5., Uberlândia, 2016. Anais..., Uberlândia, 2016. p. 665.

OECD. Pisa 2015 results: excellence and equity in education. Paris: OECD Publishing, 2016. Disponível em: <https://doi.org/10.1787/ 9789264266490-en>. Acesso em: 09 jul. 2018.

ORGANISATION FOR ECONOMIC CO-OPERATION AND DEVELOPMENT. Pisa 2012 results in focus: what 15-year-olds know and what they can do with what they know. Washington, DC: Author, 2013.

QEDU. Aprendizado dos alunos: Brasil. 2015. Disponível em: <http://www.qedu.org.br/brasil/ aprendizado >. Acessado em: 05 jul. 2018.

SARTORI, A. S. T.; DUARTE, C. G. O lúdico como uma tecnologia do desejo na educação matemática escolar. In: SIMPEMAD-SIMPÓSIO EDUCAÇÃO MATEMÁTICA EM DEBATE, 1., Joinville, 2014. Anais..., Joinville, 2014. p. 163-175.

SAVI, R.; ULBRICHT, V. R. Jogos digitais educacionais: benefícios e desafios. Revista Novas Tecnologias na Educação, Porto Alegre, v. 6, n. 2 , p. 10, 2008.

SOMMERVILLE, I.; SAWYER, P. Requirements engineering: a good practice guide. New York: John Wiley \& Sons, 1997.

TORI, R., KIRNER, C.; SISCOUTTO, R. A. Fundamentos e tecnologia de realidade virtual e aumentada. Belém, PA: SBC, 2006. 